\title{
Periodic Solutions in a Model of Pulsar Rotation
}

\author{
O. I. Bogoyavlensky \\ V.A. Steklov Mathematical Institute, The Academy of Sciences of the USSR, \\ SU-117333 Moscow, USSR
}

\begin{abstract}
A rotating rigid body with ellipsoidal cavity filled with magnetic fluid is considered as a pulsar model. Dynamical equations for the pulsar model are derived and investigated, certain integrable cases are indicated. Threeparameter sets of periodic solutions integrable in terms of elliptic functions of the time variable are obtained. A formula is derived for the period of rotation and magneto-rotational oscillations of the pulsar.
\end{abstract}

\section{Introduction}

It is now generally acknowledged [1] that pulsars (neutron stars) have a solid envelope and a liquid core which has high conductivity (the liquid is plasma) and strong frozen magnetic fields; the liquid core contains the dominating part of the pulsar mass. "Starquakes" happen periodically in pulsars, and they can be observed as glitches of the pulsar rotation period. Asynchronous rotations of the pulsar core and envelope take place during the time intervals between two such phenomena. The relaxation time for the Vela pulsar (PSR 0833-45) is $\tau \approx 6$ years, while its rotation period is $P=0.089 \mathrm{~s}$. Therefore the viscosity effects are negligible and an appropriate model of the pulsar core is that of the ideal incompressible magnetic fluid.

The model of the pulsar rotation which is proposed in the present work takes into account magnetic properties of the core and asynchronous rotation of the core and envelope (Sect. 1). The model is applicable for a finite time interval $t$, $P \ll t<\tau$, between two subsequent starquakes, where the energy losses to the viscous friction and electromagnetic radiation may be neglected.

The dynamical system describing the rotation of the pulsar model is derived in Sect. 2. It is a system of nine ordinary differential equations which are represented in a simple vector form, Eq. (2.12). This system has four first integrals $J_{k} ; J_{1}$ is the total energy of the pulsar, $J_{2}$ is the total angular momentum squared, $J_{3}$ defines the magnitude of the frozen magnetic field, and $J_{4}$ is the scalar product of the curl of the fluid velocity by the magnetic intensity vector (Sect. 3). 
The most important mathematical problem in the considered model is the existence of periodic solutions, as actually the pulsar rotation is periodic and the period is maintained with a high accuracy for a long time. It is shown in Sect. 4 that in every manifold of the first integral level $J_{i}=k_{i} k_{4}=0$ (for the domain of values of constants $k_{i}$ ) there are 12 closed trajectories of the dynamical system (2.12). These trajectories have been integrated explicitly in terms of elliptic functions of the time variable. Magneto-rotational oscillations for which the sign of the pulsar angular velocity periodically changes have been found for the present model. Such oscillations are in principle connected with the presence of magnetic fields. A formula is derived [Eq. (4.12)] expressing the minimal period of rotations and the magneto-rotational oscillations of the pulsar via its physical parameters. For the real values of the parameters the predicted period of rotation $T_{0} \approx 1 \mathrm{~s}$, which fully agrees with astrophysical data.

The dynamics of the pulsar model has some important mathematical properties: the dynamical system described by Eqs. (2.12) is a special case of Euler's equations in the space $L^{*}$ which is dual to the Lie algebra associated with the group $\mathrm{E}_{3} \times \mathrm{SO}(3)$, where $\mathrm{E}_{3}$ is the group of motions of the three-dimensional Euclidean space. In the invariant manifolds corresponding to fixed values of the integrals $J_{2}=k_{2}, J_{3}=k_{3}, J_{4}=k_{4}$ the system considered is of the Hamilton type, and its Hamiltonian is $J_{1}$. Some integrable cases are indicated in Sect. 3.

\section{The Model of the Pulsar Rotation}

The model of the pulsar dynamics is based on the following assumptions.

A. The pulsar envelope is absolutely rigid. Its liquid core has a constant density $\varrho$, and the fluid fills an ellipsoidal cavity with semi-axes $d_{1}, d_{2}, d_{3}$. The chosen reference frame $S$ is fixed to the envelope, the origin of the frame is at the center of mass of the pulsar, and the coordinate axes are parallel to the principal axes of the ellipsoid. The center of the ellipsoid 0 has coordinates $r^{1}, r^{2}, r^{3}$ in the reference frame $S$.

B. The rotation of the pulsar envelope is represented with an orthogonal matrix $Q_{1}(t)$. The motion of the fluid in the cavity is described by magnetohydrodynamical equations [2] which are

$$
\begin{aligned}
& \varrho d v / d t=-\operatorname{grad} p+(\operatorname{rot} H \times H) / 4 \pi-\varrho \operatorname{grad} \Phi, \\
& \operatorname{div} v=0, \quad \partial H / \partial t=\operatorname{rot}(v \times H), \quad \operatorname{div} H=0,
\end{aligned}
$$

where $v$ is the velocity vector, $p$ is the pressure, and $H$ is the magnetic field intensity vector; $\Phi$ is the gravitational Newton potential inside the fluid. The motion of the fluid is a motion with homogeneous deformation [3,4], and the transformation from the Lagrange coordinates $a^{k}$ to the Euler coordinates $x^{i}$ is

$$
x^{i}=\sum_{k=1}^{3}\left(F_{k}^{i} a^{k}+\left(Q_{1}\right)_{k}^{i} r^{k}\right), \quad F=Q_{1} D Q_{2}
$$

Here $Q_{2}(t)$ is an orthogonal matrix, $D_{i j}=d_{i} \delta_{i j}$; the Lagrange coordinates $a^{k}$ lie within the unit sphere, $\left(a^{1}\right)^{2}+\left(a^{2}\right)^{2}+\left(a^{3}\right)^{2} \leqq 1$. 
C. The magnetic field $H^{i}$ at the point with coordinates (1.2), is

$$
H^{i}=\sum_{k, j=1}^{3} F_{k}^{i} h_{j}^{k} a^{j}
$$

where $h_{j}^{k}$ is a constant skew-symmetrical matrix.

D. The electromagnetic field has a discontinuity at the fluid-envelope interface $\phi_{1}$. On both sides of the interface the magnetic field is tangent to the ellipsoid surface and frozen in the medium. Magnetic lines of force from the envelope are closed in the surrounding vacuum.

We will show that all the necessary boundary conditions are fulfilled at the boundary of the ellipsoidal cavity, that is the discontinuity surface. Let $H_{n}, H_{\tau}, E_{n}$, $E_{\tau}, v_{n}, v_{\tau}$ be normal and tangent components of the magnetic field, electric field, and the fluid velocity at the surface. The boundary conditions known in magnetic hydrodynamics [2] are (thermal conductivity is neglected)

$$
\begin{gathered}
\left\{E_{\tau}\right\}=0, \quad\left\{E_{n}\right\}=4 \pi \theta, \quad\left\{H_{n}\right\}=0, \quad\left\{H_{\tau}\right\}=4 \pi c^{-1}(\mathbf{i} \times \mathbf{n}) . \\
\left\{\varrho v_{n}\right\}=0, \quad\left\{s_{n}-(P \cdot \mathbf{v}) \mathbf{n}+\varrho v_{n}\left(\varepsilon+\mathbf{v}^{2} / 2\right)\right\}=0, \\
\left\{\varrho v_{n} \mathbf{v}-P \cdot \mathbf{n}-T \cdot \mathbf{n}\right\}=0, \\
\mathbf{s}=c(\mathbf{E} \times \mathbf{H}) / 4 \pi, \quad P_{i j}=-p \delta_{i j}, \quad T_{i j}=\left(H_{i} H_{j}-H^{2} \delta_{i j} / 2\right) / 4 \pi .
\end{gathered}
$$

Here $\{X\}=X_{+}-X_{-}$stands for the discontinuity of the quantity $X$ at the interface, $\theta$ is the surface charge, $\mathbf{i}$ is the surface current, $\mathbf{n}$ is the normal vector to the surface, $\mathbf{s}$ is the vector of the electromagnetic energy flux density, $P$ and $T$ are matrices with components $P_{i j}, T_{i j}$; $\varepsilon$ is the internal energy density of the fluid.

From the Eqs. (1.2), (1.3) we have $v_{n}=0, H_{n}=0$. In the approximation which is adopted in magnetic hydrodynamics $\mathbf{E}=-(\mathbf{v} \times \mathbf{H}) / c$, so $E_{\tau}=0$. Consequently, conditions (1.4) are fulfilled and determine the surface current and charge density, in the envelope $H_{n}=0, E_{\tau}=0$. Conditions (1.5) are fulfilled, as $v_{n}=0, s_{n}=0$. Since $v_{n}=0, H_{n}=0$, conditions (1.6) lead to $\left\{p+H^{2} / 8 \pi\right\}=0$. The latter condition determines the pressure from the envelope, thus it is also fulfilled in the case of the absolutely rigid envelope.

The electromagnetic field has a discontinuity at the outer vacuum-envelope interface $\phi_{2}$. The electromagnetic field in the surrounding vacuum may be, for example, the field of a magnetic dipole. It is supposed that this field rotates together with the pulsar; radiation of electromagnetic waves is not taken into account. The conditions (1.4), (1.6) determine the surface current and the pressure in the envelope at the boundary (the surface charge is $\theta=0$, because due to infinite electric conductivity of the envelope one has $E=0$ and hence $E_{n}=4 \pi \theta=0$ ). Boundary values of the magnetic field, determined on two surfaces $\phi_{1}$ and $\phi_{2}$, are matched by some magnetic field $H_{0}$ inside the envelope. The Maxwell equations inside the envelope lead to the condition that the magnetic field $H_{0}$ is frozen and to the determination of the volume current in the envelope $j=c(4 \pi)^{-1} \operatorname{rot} H_{0}$, and no other additional constraints arise.

The dynamics of the pulsar model is considered during time intervals for which the fluid viscosity and the energy loses due to electromagnetic radiation may be neglected. 


\section{Dynamics of a Rigid Body with an Ellipsoidal Cavity Filled with Magnetic Fluid}

The equation of motion (relative to the center of mass) for a rigid body having a cavity filled with magnetic fluid are equations of magnetic hydrodynamics (1.1) combined with the conservation law for the total angular momentum. Let us introduce the notations

$$
\dot{Q}_{1}=Q_{1} A, \quad \dot{Q}_{2}=-B Q_{2},
$$

and use the isomorphism between three-dimensional vectors and skewsymmetrical $3 \times 3$ matrices in space $R^{3}$,

$$
v^{i} \rightarrow V_{j k}=-\sum_{i=1}^{3} v^{i} \varepsilon_{i j k},
$$

where $v^{i}$ are the vector components and $V_{j k}$ are the matrix elements. Under this isomorphism the vector product $x \times y$ is corresponding to the commutator of the matrices, $[X, Y]=X Y-Y X$. Skew-symmetrical matrices $A, B$ are mapped to vectors with components $A^{i}, B^{i}, i=1,2,3$.

The angular momentum of the fluid in the cavity (relative to the center of mass) is (the integral is over the cavity volume)

$$
\begin{gathered}
M_{0}^{i}=\varrho \int(x \times v)^{i} d x^{1} d x^{2} d x^{3}=\sum_{j, k=1}^{3}\left(-\frac{1}{2} \varepsilon_{i j k} M_{j k}+\left(Q_{1}\right)_{j}^{i} I_{j k}^{0} A^{k}\right), \\
M=m_{1}\left(\dot{F} F^{t}-F \dot{F}^{t}\right)=m_{1} Q_{1}\left(D^{2} A+A D^{2}-2 D B D\right) Q_{1}^{t}, \\
I_{j k}^{0}=m\left(\delta_{j k} \sum_{\ell=1}^{3}\left(r^{t}\right)^{2}-r^{j} r^{k}\right), \quad m=4 \pi \varrho d_{1} d_{2} d_{3} / 3, \quad m_{1}=m / 5 .
\end{gathered}
$$

Here $m$ is the total mass of the fluid, the superscript $t$ stands for the matrix transposition, $M_{j k}$ are elements of the matrix $M$.

The total angular momentum of the system (rigid body and fluid) has the following components in the reference frame $S$ :

$$
\begin{gathered}
M^{i}=\sum_{k=1}^{3} I_{i k} A^{k}-\gamma_{i} B^{i}, \\
I_{i k}=g_{i} \delta_{i}^{k}+m_{1}^{-1}\left(I_{i k}^{0}+I_{i k}^{1}\right), \quad g_{i}=d_{j}^{2}+d_{\ell}^{2}, \quad \gamma_{i}=2 d_{j} d_{\ell},
\end{gathered}
$$

where $I_{i k}^{1}$ is the inertia tensor of the rigid envelope in the reference frame $S, i, j, \ell$ $=1,2,3$. The conservation law for the total angular momentum looks like

$$
\dot{\mathbf{M}}=\mathbf{M} \times \mathbf{A} \text {. }
$$

The last three equations of magnetic hydrodynamics in (1.1) are fulfilled identically because of the definitions (1.2) and (1.3). Turning to transformation of the first equation in (1.1), note that in the case of the ideal incompressible fluid, gravitational forces are equivalent to a redefinition of the pressure, $p_{1}=p+\varrho \Phi$, so they do not influence the dynamics of the model in view. For the motion with homogeneous deformation the effective pressure $p_{1}$ is a quadratic function of the coordinates,

$$
p_{1}=p_{0}(t)+\sum_{i, j=1}^{3}\left(p_{i j}(t) a^{i} a^{j}+p_{i}(t) a^{i}\right),
$$


where $p_{i j}(t)$ are components of a symmetrical matrix $P_{0}(t)$. Using this form of the pressure and substituting Eqs. (1.2), (1.3) in the first equation in (1.1) we conclude that this equation is equivalent to the following equations, a matrix one and a vector one,

$$
\begin{gathered}
\varrho \ddot{F}=-\left(F^{-1}\right)^{t} P_{0}+\left(\left(F^{-1}\right)^{t} h F^{t} F h+F h^{2}\right) / 4 \pi, \\
p_{i}(t)=-\sum_{k, \ell=1}^{3} F_{i}^{k}\left(Q_{1}\right)_{\ell}^{k} r^{\ell} .
\end{gathered}
$$

Introduce the notation $K_{0}=\dot{F}^{t} F-F^{t} \dot{F}$; evidently, $\dot{K}_{0}=\ddot{F}^{t} F-F^{t} \ddot{F}$ is the antisymmetrical part of the matrix $F^{t} \ddot{F}$. The symmetrical part of this matrix determines the matrix $P_{0}(t)$,

$$
2 P_{0}=-\varrho\left(F^{t} \ddot{F}+\ddot{F}^{t} F\right)+(2 \pi)^{-1} h F^{t} F h+(4 \pi)^{-1}\left(F^{t} F h^{2}+h^{2} F^{t} F\right) .
$$

Because of Eq. (2.5) we have

$$
\varrho \dot{K}_{0}=(4 \pi)^{-1}\left(h^{2} F^{t} F-F^{t} F h^{2}\right) .
$$

Using the definition (2.1) we get

$$
K_{0}=Q_{2}^{t} K Q_{2}, \quad K=D^{2} B+B D^{2}-2 D A D, \quad F^{t} F=Q_{2}^{t} D^{2} Q_{2} .
$$

By means of these formulae, Eq. (2.7) is transformed to an equivalent form,

$$
\dot{K}=[K, B]+\kappa\left[Q_{2} h^{2} Q_{2}^{t}, D^{2}\right], \quad \kappa=(4 \pi \varrho)^{-1},
$$

where the square brackets stand for the matrix commutator. With the notation $u=Q_{2} h Q_{2}^{t}$, we get, because of $(2.1)$

$$
\dot{u}=[u, B], \quad\left[u^{2}, D^{2}\right]=\left[u, u D^{2}+D^{2} u\right] .
$$

The isomorphism (2.2) maps the skew-symmetrical matrix $u$ to the vector with the components $u^{1}, u^{2}, u^{3}$, and the matrices $K$ and $w$ are mapped to vectors with the components

$$
K^{i}=g_{i} B^{i}-\gamma_{i} A^{i}, \quad w^{i}=\kappa g_{i} u^{i}, \quad i, j, k=1,2,3
$$

(no sum over $i$ !). Equations (2.4), (2.9), and (2.10), rewritten in the vector notations (2.3), (2.11), are the complete set of equations describing the dynamics of a rigid body with an ellipsoidal cavity filled with the magnetic fluid,

$$
\dot{\mathbf{M}}=\mathbf{M} \times \mathbf{A}, \quad \dot{\mathbf{K}}=\mathbf{K} \times \mathbf{B}+\mathbf{u} \times \mathbf{w}, \quad \dot{\mathbf{u}}=\mathbf{u} \times \mathbf{B} .
$$

The above equations determine completely the time evolution of the matrix $F$, so Eq. (2.6) and the second equation in (2.5) enable one to get the matrix $P_{0}(t)$ and the coefficients $p_{i}(t)$, that is to say, to calculate the pressure inside the fluid (up to an inessential additive constant).

Equations (2.12) are a generalization of the classical equations describing motion of a body with a cavity filled with the ideal incompressible fluid [5]; in the present work they are derived for the first time. The classical case corresponds to the absence of the magnetic field, it is obtained from (2.12) if $\mathbf{u}=0$. 


\section{First Integrals of the Dynamical System. The Integrable Cases}

I. The most important first integral of the dynamical system (2.12) is that corresponding to the total energy $E$ (with the constant gravitational energy excluded). It is the sum of the fluid kinetic energy $E_{1}$, the internal energy of the magnetic field $E_{2}$, and the kinetic energy of the rotation of the rigid body $E_{3}$,

$$
\begin{aligned}
& E_{1}=\int \frac{1}{2} \varrho v^{2} d x^{1} d x^{2} d x^{3}=\frac{1}{2} m_{1} \operatorname{Tr}\left(\dot{F} \dot{F}^{t}\right)+\frac{1}{2} \sum_{i, k=1}^{3} I_{i k}^{0} A^{i} A^{k}, \\
& E_{2}=\int \frac{1}{8 \pi} H^{2} d x^{1} d x^{2} d x^{3}=\frac{1}{30} \operatorname{Tr}\left(h^{t} F^{t} F h\right) d_{1} d_{2} d_{3}, \\
& E_{3}=\frac{1}{2} \sum_{i, j=1}^{3} I_{i j}^{1} A^{i} A^{j}, \quad E=E_{1}+E_{2}+E_{3} .
\end{aligned}
$$

Writing these formulae in the notations of Sect. 2, we have

$$
\begin{aligned}
2 H & =2 E / m_{1}=(\mathbf{M}, \mathbf{A})+(\mathbf{K}, \mathbf{B})+(\mathbf{u}, \mathbf{w}) \\
& =\sum_{i, k=1}^{3}\left(I_{i k} A^{i} A^{k}-2 \gamma_{i} A^{i} B^{i}+g_{i}\left(B^{i}\right)^{2}+\kappa g_{i}\left(u^{i}\right)^{2}\right), \\
g_{i} & =d_{j}^{2}+d_{k}^{2}, \quad \gamma_{i}=2 d_{j} d_{k}, \quad i, j, k=1,2,3 .
\end{aligned}
$$

Evidently, $M^{i}=\partial H / \partial A^{i}, K^{i}=\partial H / \partial B^{i}, w^{i}=\partial H / \partial u^{i}$. It is easy to verify directly that the function $J_{1}=H$ is the first integral of the system (2.12). Other three first integrals of the system are

$$
J_{2}=(\mathbf{M}, \mathbf{M}), \quad J_{3}=(\mathbf{u}, \mathbf{u}), \quad J_{4}=(\mathbf{K}, \mathbf{u}) .
$$

The integral $J_{2}$ is, up to a factor, the total angular momentum squared, $J_{3}$ is the magnetic field intensity in the Lagrange coordinates, squared, and $J_{4}$ is the scalar product of the fluid velocity curl vector by the magnetic field vector $\mathbf{h}$. All three integrals (3.3) in combination determine the six-dimensional manifold $\mathscr{M}^{6}=T\left(S^{6}\right)$ $\times S^{2}$, which is the product of the bundle tangent to the two-dimensional sphere by the two-dimensional sphere $S^{2}$.

The system (2.12) is a special case of Euler's equations [6] in the space $L^{*}$ dual to the Lie algebra $L$ which is the sum of the Lie algebra associated with the group of motions of the three-dimensional Euclidean space, $E_{3}$, and the Lie algebra of $\mathrm{SO}(3)$. The manifolds $\mathscr{M}^{6}$ are orbits of the co-adjoint representation of the Lie group $G=E_{3} \times \mathrm{SO}(3)$ in the space $L^{*}$, so the symplectic structure is determined in these manifolds in the standard manner [6]; in $\mathscr{M}^{6}$ the system (2.12) is of the Hamilton type, and its Hamiltonian is $H$.

In the case of a spherical cavity $\left(d_{1}=d_{2}=d_{3}\right)$ the magnetic field produces no effect on the system dynamics, and equations (2.12) are reduced to the usual Euler equations describing the rotations of an effective rigid body. In the case where the rigid body and the cavity have an axial symmetry,

$$
d_{1}=d_{2}, \quad r^{i}=\left(0,0, r^{3}\right), \quad I_{i k}=I_{i} \delta_{i}^{k}, \quad I_{1}=I_{2},
$$

Eqs. (2.12) have an additional first integral $J_{5}=M^{3}+K^{3}$; they are invariant under simultaneous rotations in the planes $\left(M^{1}, M^{2}\right),\left(K^{1}, K^{2}\right)$, and $\left(u^{1}, u^{2}\right)$. Therefore 
the system (2.12) at the common level of the first integrals, given in Eqs. (3.3), and of the additional integral $J_{5}$, is reduced after the factorization by this one-parameter group to a Hamiltonian system in a four-dimensional manifold, and the latter system is not integrable in general.

II. Let us consider the important case where the total angular momentum of the system is zero, $J_{2}=0$. We suppose that the center of mass is at the center of the ellipsoid $\left(r^{i}=0\right)$, and the tensor of inertia of the rigid body is diagonal, $I_{i k}^{1}=I_{i} \delta_{i}^{k}$. Then because of Eq. (2.3) we have $A^{i}=\gamma_{i} B^{i}\left(g_{i}+m_{1}^{-1} I_{i}\right)^{-1}$, and the system (2.12) is reduced to

$$
\begin{aligned}
& \dot{\overline{\mathbf{K}}}=\overline{\mathbf{K}} \times \mathbf{B}+\mathbf{u} \times \mathbf{w}, \quad \dot{\mathbf{u}}=\mathbf{u} \times \mathbf{B}, \\
& B^{i}=\partial \bar{H} / \partial \bar{K}_{i}, \quad w^{i}=\partial \bar{H} / \partial u^{i}, \quad 2 H=\sum_{i=1}^{3}\left(f_{i}^{-1} \bar{K}_{i}^{2}+\kappa g_{i} u_{i}^{2}\right), \\
& f_{i}=g_{i}-\gamma_{i}^{2}\left(g_{i}+I_{i} m_{1}^{-1}\right)^{-1} .
\end{aligned}
$$

Equations (3.4) are analogous to the classical Kirchhoff equations describing the motion of a rigid body having three symmetry planes in the ideal incompressible fluid. It is known from the theory of the Kirchhoff equations that under the Clebsch conditions [7],

$$
f_{1}\left(g_{2}-g_{3}\right)+f_{2}\left(g_{3}-g_{1}\right)+f_{3}\left(g_{1}-g_{2}\right)=0,
$$

the system (3.4) has the additional first integral

$$
J=\bar{K}_{1}^{2}+\bar{K}_{2}^{2}+\bar{K}_{3}^{2}+\kappa f_{2}\left(g_{1}-g_{3}\right) u_{1}^{2}+\kappa f_{1}\left(g_{2}-g_{3}\right) u_{2}^{2},
$$

and is therefore completely integrable.

We shall show that for any magnitude of semi-axes of the ellipsoidal cavity, $d_{1}$, $d_{2}, d_{3}$, there exists a two-parameter family of values of the rigid body inertia tensor $I_{i}$ for which relation (3.5) holds; that is to say the system dynamics is integrable at the level $J_{2}=0$. Put $d_{3}>d_{1}>d_{2}$, and introduce the following notations:

$$
\begin{aligned}
& \beta_{i}=1+m_{1}^{-1} I_{i} g_{i}^{-1}>1, \quad x_{1}=d_{1} d_{3}^{-1}, \quad x_{2}=d_{2} d_{3}^{-1}, \quad x_{2}<x_{1}<1, \\
& \alpha_{1}=2 x_{2}\left(1+x_{1}^{2}\right)^{-1}, \quad \alpha_{2}=2 x_{1}\left(1+x_{2}^{2}\right)^{-1}, \quad \alpha_{3}=2 x_{1} x_{2}\left(x_{1}^{2}+x_{2}^{2}\right)^{-1} .
\end{aligned}
$$

We get from Eq. (3.4) that $f_{i}=g_{i}\left(1-\alpha_{i}^{2} \beta_{i}^{-1}\right)$. After substitution of (3.7) and a simple transformation, Eq. (3.5) is reduced to the form

$$
\frac{x_{1}^{2}\left(1-x_{1}^{2}\right)}{\beta_{2}\left(1+x_{1}^{2}\right)}+\frac{x_{2}^{2}\left(x_{2}^{2}-1\right)}{\beta_{1}\left(x_{2}^{2}+1\right)}+\frac{x_{1}^{2} x_{2}^{2}\left(x_{1}^{2}-x_{2}^{2}\right)}{\beta_{3}\left(x_{1}^{2}+x_{2}^{2}\right)}=0 .
$$

In view of this equation, we have $\beta_{1}>0$ for two arbitrary parameters $\beta_{2}, \beta_{3}>0$ and at $0<x_{2}<x_{1}<1$. The solutions of this equation admit the transformation $\beta_{i} \rightarrow L \beta_{i}$, so we get the two-parameter solution with $\bar{\beta}_{i}=L \beta_{i}>1$ if $L$ is large enough. Then the corresponding components of the inertia tensor of the rigid body, $I_{i}$, are found from Eq. (3.7). In particular, Eq. (3.8) has solutions for which $x_{1} \approx x_{2} \approx 1$ and $\beta_{1} \approx \beta_{2} \approx \beta_{3}$, and the necessary conditions $I_{i}<I_{j}+I_{k}$ are also fulfilled in this case for large $L$.

The familiar integrable case found by S.A. Chaplygin [8] for the Kirchhoff equations does also belong to the system considered. Under the conditions

$$
f_{1}=f_{2}=2 f_{3}, \quad g_{3}=\frac{1}{2}\left(g_{1}+g_{2}\right), \quad J_{4}=0,
$$


Eqs. (3.4) have an additional first integral,

$$
J_{5}=\left(\left(K_{2}^{2}-K_{1}^{2}\right) f_{3}^{-1}+\kappa\left(d_{1}^{2}-d_{2}^{2}\right) u_{3}^{2}\right)^{2}+4 f_{3}^{-2} K_{1}^{2} K_{2}^{2},
$$

and the system is therefore completely integrable. After substitution of Eqs. (3.4), (3.7) it is not difficult to see that Eqs. (3.9) have a three-parameter family of solutions $d_{i}, I_{k}$ satisfying all the necessary conditions.

\section{Periodic Solutions}

I. For periodic rotations in the pulsar model the matrices $Q_{1}(t), Q_{2}(t)$ are periodic functions of the time variable which have identical periods. Closed trajectories of the system (2.12) are corresponding to such solutions; inversely, if the closed trajectories of the system (2.12) form a three-dimensional set, it has an everywhere dense subset corresponding to periodic rotations $Q_{1}(t)$ which leads to periodic variations of the external electromagnetic field of the pulsar.

It will be seen that under the conditions $I_{i k}=\left(g_{i}+I_{i}\right) \delta_{i k}$ and $J_{4}=0$ there are 12 closed trajectories of the system (2.12) in the open set of the level surfaces for the first integrals $J_{i}=k_{i}$.

For $I_{i k}=\left(g_{i}+I_{i}\right) \delta_{i k}$ the integral $J_{1}=H$ is

$$
\begin{aligned}
2 J_{1} & =\sum_{i=1}^{3}\left(a_{i} M_{i}^{2}+2 c_{i} M_{i} K_{i}+b_{i} K_{i}^{2}+\kappa g_{i} u_{i}^{2}\right), \\
a_{i} & =g_{i} s_{i}, \quad c_{i}=\gamma_{i} s_{i}, \quad b_{i}=\left(g_{i}+I_{i}\right) s_{i}, \quad s_{i}=\left(\left(g_{i}+I_{i}\right) g_{i}-\gamma_{i}^{2}\right)^{-1} .
\end{aligned}
$$

At the level $J_{4}=0$ the system (2.12) has three invariant submanifolds $V_{k}^{4}: u_{k}=M_{i}=M_{j}=K_{i}=K_{j}=0(i, j, k=1,2,3)$. In the manifold $V_{1}^{4}$ Eqs. (2.12) and the integrals in Eqs. (3.2) and (3.3) are written as

$$
\begin{aligned}
\dot{K}_{3} & =\kappa\left(g_{2}-g_{1}\right) u_{1} u_{2}, \quad \dot{u}_{1}=u_{2} B_{3}, \quad \dot{u}_{2}=-u_{1} B_{3}, \quad \dot{M}_{3}=0, \\
2 J_{1} & =a_{3} M_{3}^{2}+2 c_{3} M_{3} K_{3}+b_{3} K_{3}^{2}+\kappa g_{1} u_{1}^{2}+\kappa g_{2} u_{2}^{2}, \\
J_{2} & =M_{3}^{2}, \quad J_{3}=u_{1}^{2}+u_{2}^{2}, \quad B_{3}=c_{3} M_{3}+b_{3} K_{3} .
\end{aligned}
$$

The level surface for the integral $J_{2}=k_{2}$ contains two components $M_{3}=\varepsilon k_{2}^{1 / 2}$, $\varepsilon= \pm 1$. In each component the manifold given by the integrals $J_{1}=k_{1}, J_{3}=k_{3}$ is the intersection of an ellipsoid $\left(J_{1}=k_{1}\right)$ and a cylinder $\left(J_{3}=k_{3}\right)$ having a common axis $K_{3}$; it either consists of two closed trajectories of the system (4.2), or is empty (the number of these closed trajectories is equal for both components of the manifold, $M_{3}=\varepsilon k_{2}^{1 / 2}$ ). The total number of closed trajectories in three invariant submanifolds $V_{k}^{4}$ depends on the relation between the quantities $J_{1}, J_{2}, J_{3}$; this number is $12,8,4$ or zero, and there are exactly 12 closed trajectories for $2 J_{1}>J_{2} \cdot \max \left(a_{i}-c_{i}^{2} / b_{i}\right)+\kappa J_{3} \max \left(g_{i}\right)$.

The closed trajectories describe the pulsar rotation around a fixed axis. The maximal number of such trajectories ( 4 for every one of three axes) is associated with two possible directions of the pulsar total angular momentum and two possible directions of the rotation of the liquid core with respect to the envelope.

After the substitution $K_{3}=\left(B_{3}-c_{3} M_{3}\right) / b_{3}$, Eqs. (4.2) acquire the form of the classical Euler's equations,

$$
\dot{B}_{3}=-\omega u_{1} u_{2}, \quad \dot{u}_{1}=u_{2} B_{3}, \quad \dot{u}_{2}=-u_{1} B_{3},
$$


where $\omega=\kappa b_{3}\left(g_{1}-g_{2}\right)$. Let us calculate the period of the closed trajectories for the system (4.2) and (4.3). The integrals of the system (4.3) are

$$
\ell=B_{3}^{2}+\omega u_{1}^{2}, \quad J_{3}=u_{1}^{2}+u_{2}^{2} .
$$

Let $d_{2}>d_{1}$, then $\omega=\kappa b_{3}\left(d_{2}^{2}-d_{1}^{2}\right)>0$. Expressing $B_{3}$ and $u_{2}$ via $u_{1}$ by means of $(4.4)$ and substituting them into (4.3) we obtain

$$
\dot{u}_{1}=\left(\left(J_{3}-u_{1}^{2}\right)\left(\ell-\omega u_{1}^{2}\right)\right)^{1 / 2} .
$$

Solutions of the equations of this type as known [9] are

$$
u_{1}=(\ell / \omega)^{1 / 2} \operatorname{sn}(\tau), \quad \tau=\left(\omega J_{3}\right)^{1 / 2}\left(t-t_{0}\right),
$$

where $\operatorname{sn} \tau$ is Jacobi's elliptic function corresponding to the parameter $k^{2}=\ell /\left(\omega J_{3}\right)$. Putting the result (4.6) into Eq. (4.4), we get

$$
B_{3}=\ell^{1 / 2} \operatorname{cn} \tau, \quad u_{2}=J_{3}^{1 / 2} \mathrm{dn} \tau .
$$

The period of the elliptic functions presented in Eqs. (4.6) and (4.7) is given by the expression

$$
T=4\left(\omega J_{3}\right)^{-1 / 2} \int_{0}^{\pi / 2}\left(1-k^{2} \sin ^{2} \alpha\right)^{-1 / 2} \mathrm{~d} \alpha .
$$

This is the period of the closed trajectories for the system (4.2) and (4.3).

II. Let us find the magnitude of the period, $T$, which appears in the models of the real pulsars, that is for $d_{1} \approx d_{2} \approx d_{3} \approx R$ and at constant $J_{3}, I_{3}, \varrho$. The function $T$ in Eq. (4.8) attains the minimal value, $T_{m}$, at $k=\ell=0$; in other words, for small oscillations taking place in a vicinity of the axis $u_{2}\left(B_{3}=u_{1}=0\right)$, thereby $T_{m}=2 \pi\left(\omega J_{3}\right)^{-1 / 2}$. Asymptotically, such oscillations are $(\ell \ll 1)$

$$
u_{1}=\ell^{1 / 2} \sin \left(\omega^{1 / 2} u_{2}^{0}\left(t-t_{0}\right)\right), \quad B_{3}=(\ell \omega)^{1 / 2} \cos \left(\omega^{1 / 2} u_{2}^{0}\left(t-t_{0}\right)\right), \quad u_{2}=u_{2}^{0} .
$$

After the substitution of Eqs. (4.1) and (2.3) into Eq. (4.7) we get for $k \ll 1$,

$$
\begin{gathered}
T_{m}=2 \pi\left(4 \pi \varrho / J_{3}\right)^{1 / 2} K\left(d_{1}, d_{2}, I_{3}\right), \\
K=\left(d_{1}^{2}+d_{2}^{2}+I_{3}\right)^{-1 / 2}\left(d_{2}^{2}-d_{1}^{2}+I_{3}\left(d_{1}^{2}+d_{2}^{2}\right)\left(d_{2}^{2}-d_{1}^{2}\right)^{-1}\right)^{1 / 2}, \quad I_{3}=m_{1}^{-1}\left(I_{3}^{0}+I_{3}^{1}\right) .
\end{gathered}
$$

The function $K$ attains its maximum $K_{m}$ at

$$
d_{2}^{2}-d_{1}^{2}=\left(I_{3}\left(d_{1}^{2}+d_{2}^{2}\right)\right)^{1 / 2}, \quad K_{m}=2^{1 / 2}\left(I_{3}\left(d_{1}^{2}+d_{2}^{2}\right)\right)^{1 / 4}\left(d_{1}^{2}+d_{2}^{2}+I_{3}\right)^{-1 / 2} .
$$

Hence we obtain the minimal value of the period

$$
T_{0}=4 \pi^{3 / 2}\left(2 \varrho / J_{3}\right)^{1 / 2}\left(I_{3}\left(d_{1}^{2}+d_{2}^{2}\right)\right)^{1 / 4}\left(d_{1}^{2}+d_{2}^{2}+I_{3}\right)^{-1 / 2} .
$$

For the real pulsars we have [1]: $d_{1} \approx d_{2} \approx d_{3} \approx R \sim 10^{6} \mathrm{~cm}$, the matter density in the liquid core is $\varrho \sim 10^{14} \mathrm{~g} / \mathrm{cm}^{3}$, the matter density in the envelope is $\varrho_{1} \sim 10^{8} \mathrm{~g} / \mathrm{cm}^{3}$, the envelope thickness is $r \sim 10^{4} \mathrm{~cm}$, the magnetic field intensity at the pulsar surface is $|H| \sim 10^{12}$ Gs (all the numerical values are presented with an accuracy up to an order of magnitude). According to the definition in (1.3), the maximal magnitude of the magnetic field intensity at the surface of the ellipsoidal cavity is given by the formula $|H|=R|h|$. The definition $u=Q_{2} h Q_{2}^{t}$ leads to $J_{3}=|u|^{2}=|h|^{2}$, so $J_{3}^{1 / 2}=|H| / R$. If $r \ll R$ the interia tensor of the envelope is

$$
I_{i k}^{1}=\frac{8}{3} \pi R^{4} r \varrho_{1} \delta_{i k} \text {. }
$$


It is natural to assume that the center of mass of the pulsar is near the center of the ellipsoidal cavity, and $\left|r^{i}\right|<R\left(2 r \varrho_{1} / R \varrho\right)^{1 / 2}$, then $\left|I_{i k}^{0}\right|=\gamma\left|I_{i k}^{1}\right|$, where $\gamma<1 \quad$ [cf. Eq. (2.3)]. Putting into Eq. (4.11) the corresponding expression for the relevant component of the interia tensor,

$$
I_{3}=m_{1}^{-1}\left(I_{3}^{0}+I_{3}^{1}\right)=10(1+\gamma) \operatorname{Rr} \varrho_{1} / \varrho,
$$

we obtain finally

$$
T_{0}=8 \pi^{3 / 2}(5(1+\gamma) / 4)^{1 / 4} \varrho^{1 / 2} R H^{-1}\left(r \varrho_{1} / R \varrho\right)^{1 / 4} .
$$

Besides, we have $d_{2}=d_{1}\left[1+\left(5(1+\gamma) r \varrho_{1} / R \varrho\right)^{1 / 2}\right]$ because of Eq. (4.10). Using the numerical estimates presented above, we get $d_{2}=d_{1}\left(1+(5(1+\gamma))^{1 / 2} 10^{-4}\right)$, $T_{0}=5 \mathrm{~s}$. The obtained value of $T_{0}$ is a reasonable approximation of the period $T=3.75 \mathrm{~s}$ that is known for the pulsar PSR 0527. Having in mind the inaccuracy in numerical magnitudes of all the quantities presented in Eq. (4.12), this estimate for the minimal period of the pulsar rotation may be considered as being in a satisfactory agreement with the available astrophysical data. Putting, for instance, $H=5 \cdot 10^{12}$ Gs (this estimate is quite likely) we get $T_{0} \sim 1 \mathrm{~s}$; this value of the period is fairly close to the data for a number of pulsars, e.g. PSR $0628(T=1.24 \mathrm{~s})$, PSR $1133(T=1.19 s)$ and others [1].

III. Trajectories of the system (4.3) satisfying the condition

$$
\left(d_{1}^{2}+d_{3}^{2}\right)^{-1} J_{0}>J_{3}>\left(d_{2}^{2}+d_{3}^{2}\right)^{-1} J_{0}, \quad J_{0}=\left(2 J_{1}-\left(a_{3}-c_{3}^{2} / b_{3}\right) J_{2}\right) \kappa^{-1}
$$

are encircling the $u_{2}$ axis $\left(B_{3}=u_{1}=0\right)$, as well as the trajectories of Eq. (4.8). For such trajectories the matrix $Q$ is invariable for the period of a single oscillation, as $\dot{Q}_{2}=-B_{0} Q_{2}$, and $\oint B_{3} d t=0$, while the matrix $Q_{1}(t+T) Q_{1}^{-1}(t)$ determines the rotation around the $x_{3}$ axis by the angle $\Delta \varphi=T M_{3} / I_{3}$. The condition $\Delta \varphi=2 \pi p / q$ (where $p$ and $q$ are integer) determines the magnitude of the angular momentum, $|\bar{M}|=m_{1}\left|M_{3}\right|=2 \pi p m_{1} I_{3} / q T$, providing the exactly periodic pulsar rotation (with the period of $q T$ ).

If $J_{3} \kappa \min \left(g_{i}\right)<J_{1}<J_{3} \kappa \max \left(g_{i}\right)$ and $J_{2} \ll 1$, there are 8 closed trajectories at the surface $J_{i}=k_{i}$, and the quantities $K_{3}, B_{3}, A_{3}=a_{3} M_{3}+c_{3} K_{3}$ have alternating signs along the trajectories. Trajectories of this type describe nonmonotonous pulsar rotations around the $x_{3}$ axis, in the process of which the angular velocities of the envelope and of the internal rotation of the fluid change their signs periodically [a particular case of this type are the oscillations described by Eq. (4.9) for $\left.\left|M_{3}\right|<(\omega \ell)^{1 / 2} c_{3}\left(a_{3} b_{3}-c_{3}^{2}\right)^{-1}\right]$. Motions of this kind are possible only in the presence of an internal magnetic field and for $d_{1} \neq d_{2}$.

Magneto-rotational oscillations of the incompressible fluid in the case of the cylindrical symmetry (the object is infinite in the $x_{3}$ direction) have been investigated in [10]. The existence of some periodical trajectories for the system (2.12) can be established using the results obtained by Novikov [11]. For $J_{2}=0$ the system (2.12) is reduced to the Kirchhoff equations, so there are at least 2 closed trajectories for every level of the integrals $J_{3}, J_{4}, J_{1}>E\left(J_{3}, J_{4}\right), J_{2}=0$, as it is shown in [11].

IV. Important solutions are also those having the minimal total energy $J_{1}$ in the manifold $\mathscr{M}^{6}$ corresponding to fixed values of the integrals $J_{2}=k_{2}, J_{3}=k_{3}$, 
$J_{4}=k_{4}$. As the energy $J_{1}$ is positive definite, such solutions do exist for any manifold of levels of the integrals $J_{2}, J_{3}, J_{4}$; they correspond to stationary points of the system (2.12). At the stationary points one has

$$
\mathbf{A}=\lambda \mathbf{M}, \quad \mathbf{B}=\alpha \mathbf{u}, \quad \mathbf{w}=\alpha \mathbf{K}+\beta \mathbf{u} .
$$

After substitution of Eqs. (2.11) these conditions are reduced to

$$
\begin{gathered}
u^{i}=\alpha \lambda z_{i} M_{i}, \quad K_{i}=\left(\kappa g_{i}-\beta\right) \lambda z_{i} M_{i}, \quad z_{i}=\gamma_{i}\left(\left(\alpha^{2}-\kappa\right) g_{i}+\beta\right)^{-1}, \\
\lambda^{-1} M_{i}=\sum_{k=1}^{3} J_{i k} M_{k}, \quad J_{i k}=I_{i k}-\gamma_{i} \alpha^{2} z_{i} \delta_{i k} .
\end{gathered}
$$

Thus to find the stationary points one has to calculate the eigenvalues and eigenvectors of the matrix with elements $J_{i k}$. The rigid-body rotation $(\alpha=0)$ and the purely internal rotation of the fluid $(\lambda=0)$ take place only for a degenerate set of the singular points lying in a two-parameter set of the manifolds $\mathscr{M}^{6}$. In the general case of Eqs. (4.13) and (4.14) the matrices $Q_{1}(t)$ and $Q_{2}(t)$ describe periodic rotations with the periods $T_{1}$ and $T_{2}$. If the periods $T_{1}$ and $T_{2}$ are commensurate, the solution is exactly periodic.

\section{References}

1. Dyson, F.J.: Neutron stars and pulsars. In: Lectures 1970. Fermi, E. (ed.). Rome: Accademia Nazionale dei Lincei 1971

2. Kulikovsky, A.G., Lyubimov, G.A.: Magnetic hydrodynamics. Moscow: Nauka 1962 (in Russian)

3. Kulikovsky, A.G.: On motions with homogeneous deformation in magnetic hydrodynamics. Dokl. Akad. Nauk USSR 120, 984-986 (1958)

4. Bogoyavlensky, O.I.: Methods of qualitative theory of dynamical systems in astrophysics and gas dynamics (in Russian). Moscow: Nauka 1980; English translation is published by Springer, Berlin, Heidelberg, New York 1985

5. Moiseev, N.N., Rumyantsev, V.V.: Dynamics of body with cavities containing fluid (in Russian). Moscow: Nauka 1965

6. Arnold, V.I.: Mathematical methods of classical mechanics. Moscow: Nauka 1974 (in Russian)

7. Clebsch, A.: Über die Bewegung eines Körpers in einer Flüssigkeit. Math. Ann. 3, 238-262 (1871)

8. Chaplygin, S.A.: A new particular solution of the problem of motion of a rigid body in fluid (in Russian). Proc. Dept. Phys. Sci., Imp. Soc. Naturalists 9, 3-12 (1902)

9. Erdelyi, A., et al.: Higher transcendental functions, Vol. III. New York: McGraw-Hill 1955

10. Bisnovatyi-Kogan, G.S., Popov, Yu.P., Samochin, A.A.: The magnetohydrodynamical rotational model of supernova explosion. Astrophys. Space Sci. 41, 321-356 (1976)

11. Novikov, S.P.: Variational methods and periodical solutions of the Kirchhoff-type equations. II. Funkts. Anal. Prilozh. 15, 37-52 (1981)

Communicated by J. Mather

Received January 16, 1984; in revised form July 15, 1985 
